\title{
Nuclear re-interaction effects in quasi-elastic neutrino nucleus scattering
}

\author{
G. Co', C. Bleve, I. De Mitri, and D. Martello \\ Dipartimento di Fisica, Università di Lecce, \\ and \\ Istituto Nazionale di Fisica Nucleare sez. di Lecce, \\ I-73100 Lecce, Italy
}

\begin{abstract}
The quasi-elastic $\nu$-nucleus cross section has been calculated by using a Fermi gas model corrected to consider the re-scattering between the emitted nucleon and the rest nucleus. As an example of the relevance of this effect we show results for the muon production cross section on ${ }^{16} \mathrm{O}$ target.
\end{abstract}

The construction and the planning of new experiments with the objective of detecting neutrinos, have raised great attention on the $\nu$-nucleus interaction. From the nuclear physics point of view, new $\nu$ nucleus data offer the opportunity to further test the knowledge of the nuclear structure and, perhaps, to reveal new nuclear effects. From the astrophysics point of view, a better determination of the $\nu$-nucleus cross section would help to improve the understanding of many phenomena, like star burning, element production, supernova explosion and cooling.

Also from the point of view of an elementary particle physicist, interested in revealing the properties of the neutrinos, either coming from natural or artificial sources, the knowledge of the $\nu$-nucleus interaction is important. The reaction between neutrinos and nuclei is the basic physical process upon which many detectors are built. The understanding of their sensitivity to the neutrinos properties and the evaluation of the neutrino fluxes strongly depend on a precise knowledge of the $\nu$-nucleus cross section.

Many Monte Carlo codes simulating detector responses, describe the $\nu$-nucleus cross section by folding the free $\nu$-nucleon cross section with a Fermi gas distribution. In this model, due to Smith and Moniz [1], the effects of the Fermi motion and of the Pauli exclusion principle are taken into account. On the other hand, many other nuclear effects are neglected. The Fermi gas model considers the nucleus as an infinite system of non-interacting nucleons. Therefore all the phenomena related to the nuclear surface and to the interaction between nucleons are not considered. Because of its simplicity the Smith and Moniz model is rather popular, however its intrinsic approximations have never been thoroughly tested.

In the present contribution we analyze one of the approximations of the Fermi gas model, specifically the one neglecting the re-interaction between the emitted nucleon and rest nucleus. We have developed a model to consider this effect. Details can be found in ref. [2]. We present here results for the muon production on the ${ }^{16} \mathrm{O}$ nucleus. They have been calculated for the kinematic conditions suggested as benchmark by the conference organizers.

We have already remarked that the range of applicability of the Fermi gas model in treating nuclear excitations is restricted to situations where the surface effects can be neglected. For this reason the Fermi gas model can be reliably used only in the quasi-elastic region. Roughly speaking, this region can be identified with a range of nuclear excitation energies from 50 to $300 \mathrm{MeV}$.

The validity of the infinite system approximation in the description of the quasi-elastic excitation has been studied in ref. [3] by comparing electromagnetic responses calculated within the Fermi gas 

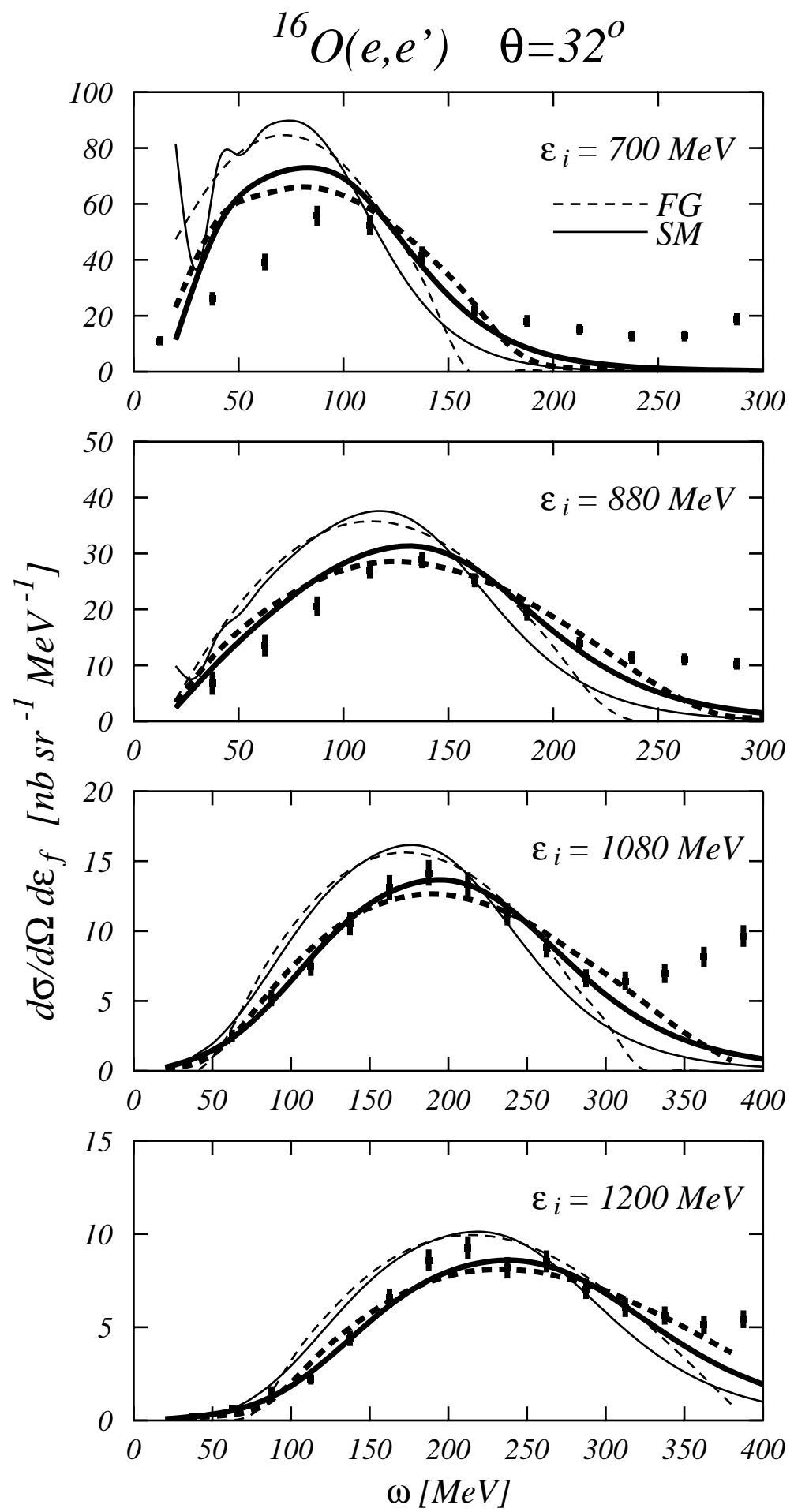

Figure 1: Comparison between Fermi gas (FG) and Continuum Shell model (SM) results. We have indicated with $\epsilon_{i}$ the initial electron energy, and with $\omega$ the missing energy, corresponding in our model with the nuclear excitation energy. The lower lines have been obtained by including the Final State Interaction. Data from ref. [1]. 
model and the Continuum Shell model. From the physics contents these two models differ only because the Continuum Shell model considers the finite nuclear dimensions. In ref. [3] it is shown that a good agreement between the Fermi gas and the Continuum Shell model results can be obtained when an appropriate value of the Fermi momentum is used. It is also shown that this is a much better prescription than the commonly used Local Density Approximation.

The thin lines of fig. 1 1 show the electron scattering cross sections on the ${ }^{16} \mathrm{O}$ nucleus calculated with the two different models. We used the value of the Fermi momentum of $216 \mathrm{MeV} / \mathrm{c}$ obtained with the average momentum prescription presented in ref. [3]. The agreement between the results of the two calculations improves with increasing the energy of the electron.

In the figure, the agreement of the thiner lines with the experimental data [ [4] is rather poor. This is a common feature of all the calculations done in the quasi-elastic region with nuclear models neglecting the re-interaction between the emitted nucleon and the rest nucleus [5]. To include this effect, commonly called Final State Interaction (FSI), we used the model developed in ref. [6].

In this model the response containing the FSI can be expressed in terms of folding integral of the response without FSI:

$$
\begin{aligned}
& S^{F S I}(|\mathbf{q}|, \omega)= \\
& \int_{0}^{\infty} d E S^{0}(|\mathbf{q}|, E)[\rho(E, \omega)+\rho(E,-\omega)]
\end{aligned}
$$

where the folding function is:

$$
\rho(E, \omega)=\frac{1}{2 \pi} \frac{\Gamma(\omega)}{[E-\omega-\Delta(\omega)]^{2}+[\Gamma(\omega) / 2]^{2}}
$$

The two functions $\Delta$ and $\Gamma$ represent the real and imaginary part of the so-called spreading width, which takes into account nuclear excitations beyond those considered in mean-field models, like the Fermi gas and the Shell models. A microscopic evaluation of this spreading width [7] has shown that, in the quasi-elastic region, its effects can be rather well described in terms of an optical potential. For this reason, as it is commonly done $[5$, 66, we fixed the $\Delta$ and $\Gamma$ functions as volume integrals of optical potentials reproducing elastic nucleon-nucleus cross sections data.

The thick curves of fig. 11 have been obtained from the thin lines by applying the procedure above outlined. The maxima of the mean field responses are lowered, and part of the strength is shifted towards higher excitation energies. The shift of the position of the maxima is due to the presence, in our model, of an effective nucleon mass smaller than the bare nucleon mass. This effective mass takes into account the non locality of the nuclear mean field.

It is evident from the figure, that the inclusion of the FSI largely improves the agreement with the data. The features we have discussed are not typical of the specific example considered. The quasi-elastic electron scattering data can be reproduced only when the FSI is considered [5].

From the above considerations it appears evident that a realistic description of the $\nu$-nucleus interaction in the quasi-elastic region requires the treatment of the FSI. Our calculations of the $\nu$-nucleus cross section have been done by using the FSI model above described [2]. As eq. (11) indicates, we need a mean field response. We have used the Fermi gas response of the Smith and Moniz approach implemented as in ref. [8].

The double differential cross section for the ${ }^{16} \mathrm{O}\left(\nu_{\mu}, \mu\right){ }^{16} \mathrm{~F}$ reaction is shown in fig. 2 as a function of the emitted muon energy for three values of the neutrino energy. The emission angle of the muon has been fixed at $30^{\circ}$. The full lines show the result obtained with a bare Fermi gas calculations, while the dashed lines contain the effects of the FSI.

A first observation is that for $\mathrm{E}_{\nu}=5.0 \mathrm{GeV}$ the FSI effects are negligible. The only difference between the two curves is a small shift of the position of the peak due to the presence of the effective nucleon mass. For this reason henceforth we shall neglect the results of the $5.0 \mathrm{GeV}$ calculations.

The FSI effects are instead rather important for the other two energy values. At $0.5 \mathrm{GeV}$ the Fermi gas response still shows the linear behaviour at high muon energies (small nuclear excitation energies). 


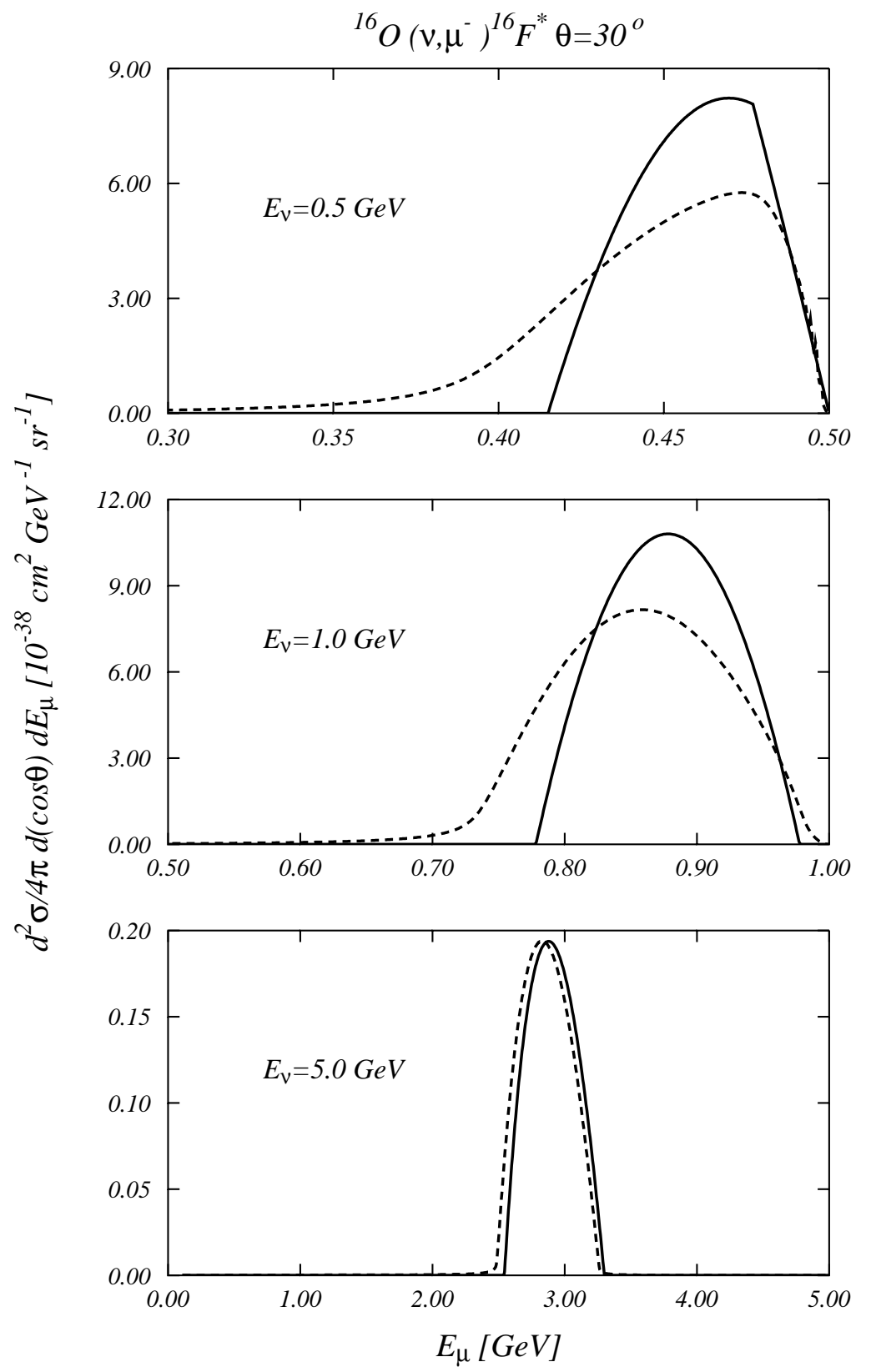

Figure 2: Double differential $\nu$-nucleus cross section as a function of the emitted muon energy for three values of the neutrino energy. The full lines have been calculated with the Fermi gas model and the dashed lines include the FSI effects. 


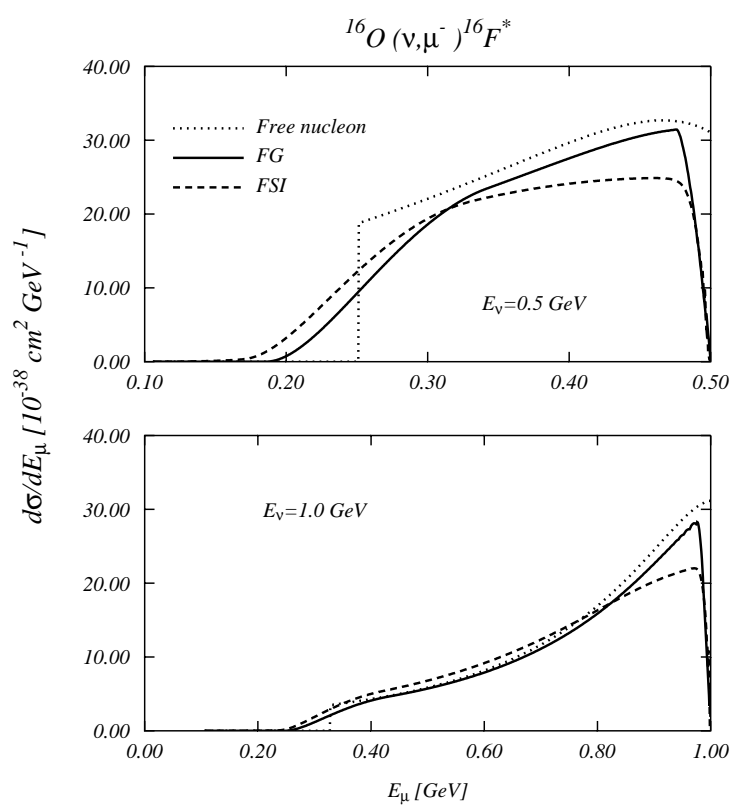

Figure 3: Cross section integrated on the muon emission angle as a function of the muon energy.

This is a signature of the fact that, under these kinematic conditions, some of the particle-hole transitions are Pauli blocked. The linear behaviour is no longer in the $1 \mathrm{GeV}$ neutrinos cross section.

In both cases the FSI strongly modifies the responses, decreasing their values in the peak region and shifting strength in the forbidden region. The FSI increases the cross section at small muon energies and decreases it at higher energies.

These modifications of the nuclear response have consequences on the integrated cross sections. In fig. 3 we show the cross sections integrated with respect to the muon emission angle, as a function of the muon energy. Together with the Fermi gas and FSI results we also show the free nucleon cross section.

The differences between the various calculations are more evident for $0.5 \mathrm{GeV}$ case. The free nucleon cross section has a sharp energy threshold. When the nucleon is embedded in the nuclear medium this sharp behaviour of the cross section is smoothed, and the cross sections is different from zero also in sub-threshold region. At high values of the muon energy (low excitation energy) the effect of the Pauli blocking is clearly visible in the nuclear cross sections. As expected, the inclusion of the FSI lowers the Fermi gas cross section at high muon energies and increases it at lower energies values. These effects become relatively smaller with increasing neutrino energy.

In fig. A we show the neutrino cross section integrated over the muon energy as a function of the emission angle. For neutrinos of 1.0 and $0.5 \mathrm{GeV}$ the curves with and without FSI are rather similar. They are instead remarkably different for $0.2 \mathrm{GeV}$.

This result indicates that the effect of the FSI in fig. 2 is predominantly a redistribution of the strength, without loss or increase of it. In that figure, the integrals of the cross sections with and without FSI are almost identical. Indeed if the $\Gamma$ and $\Delta$ functions are independent of $\omega$ these integrals would be identically the same. In the region for $\omega>50 \mathrm{MeV}$ the $\Gamma$ and $\Delta$ functions are almost constant. For neutrino energies above $0.5 \mathrm{GeV}$ the major weight in the integral comes from the region of constant $\Gamma$ and $\Delta$. This explain the result of the two lower panels of fig. 6 . The situation is rather different for $\mathrm{E}_{\nu}=0.2 \mathrm{GeV}$ as is seen in the upper panel of the figure.

We have shown that the FSI produces relevant modifications on the quasi-elastic double differential $\nu_{\mu}$-nucleus cross section. The main effect is a redistribution of the strength, shifted from the peak position 

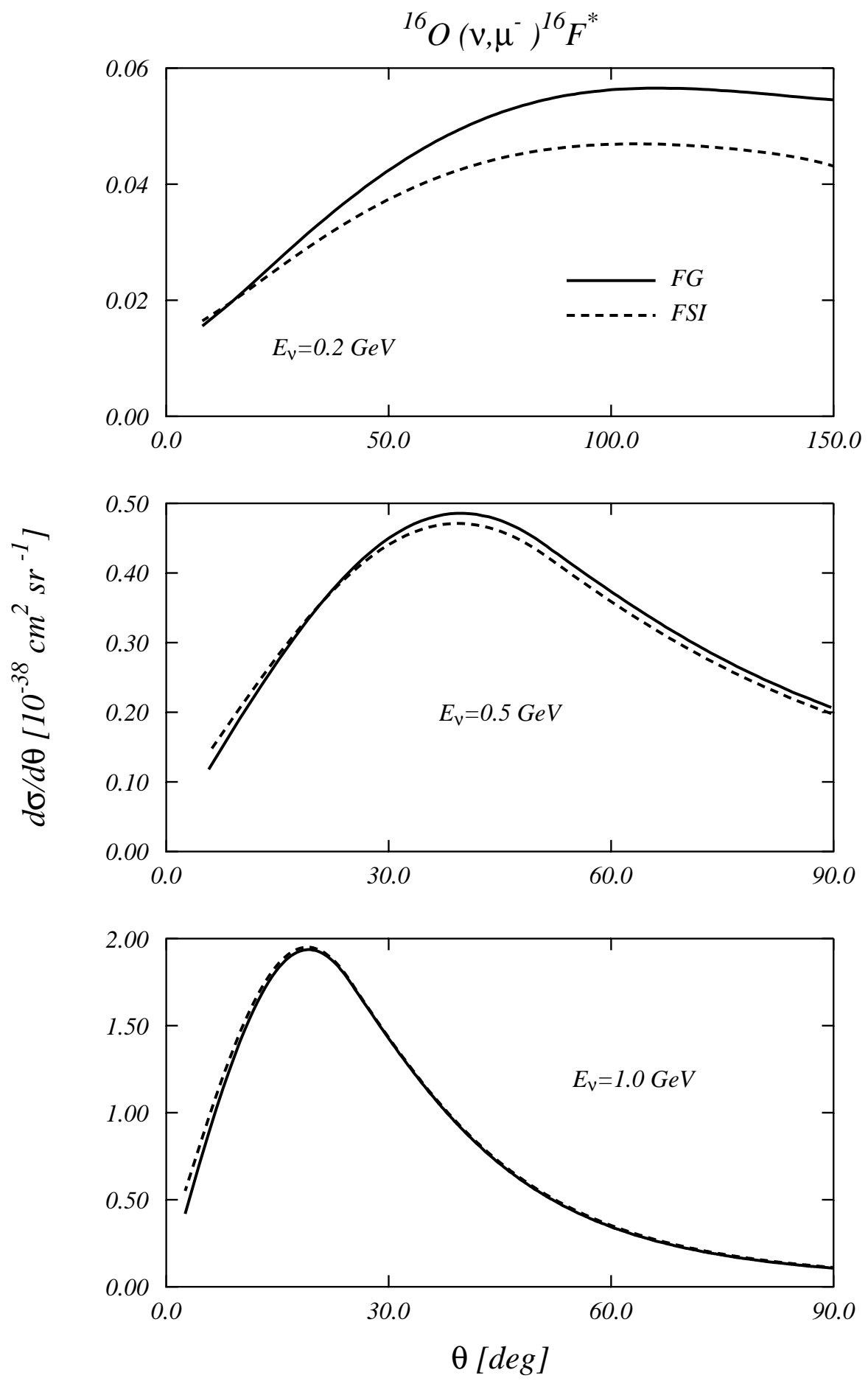

Figure 4: Cross sections integrated on the energy of the emitted muon as a function of the scattering angle. 
towards lower muon energies. The FSI effects become smaller with increasing neutrino energy and they are negligible for $5.0 \mathrm{GeV}$ neutrinos. The inclusion of the FSI is a necessary modification of the commonly used Fermi gas model, and more in general of any mean-field model, in order to have a more realistic description of the neutrino nucleus interaction. On the other hand, if the cross section is integrated on the energy of the emitted muon the FSI effects are appreciable only for rather low neutrino energies (we have shown one example of the $0.2 \mathrm{GeV}$ data).

We have presented results for the muon neutrinos and the ${ }^{16} \mathrm{O}$ nucleus. Our conclusions, however, are more general, because the FSI interactions effects are intrinsic to the nuclear excitation modes, and

independent of the excitation probe. The FSI effects above described, are present also in electron neutrino and tau neutrino reactions and for any nuclear system.

\section{References}

[1] R.A. Smith and E.J. Moniz, Nucl. Phys. B 43 (1972) 605.

[2] C. Bleve et al. , Astr. Phys. 16 (2001) 145.

[3] J.E. Amaro, A.M. Lallena and G. Co', Int. Jour. Mod. Phys. E 3 (1994) 735; E. Bauer and G. Co', Jour. Phys. G 27 (2001) 1813.

[4] M. Anghinolfi et al. , Nucl. Phys. A 602 (1996) 405.

[5] J.E. Amaro, G. Co' and A.M. Lallena, in Electromagnetic response functions in nuclei, p. 63, R. Cenni ed., Nova Science Publisher, New York, 2001.

[6] G. Co', K.F. Quader, R.D. Smith and J. Wambach, Nucl. Phys. A 485 (1988) 61.

[7] S. Drożdż. G. Co', J. Wambach and J. Speth, Phys. Lett. B 185 (1987) 287.

[8] P. Lipari, M. Lusignoli and F. Sartogo, Phys. Rev. Lett. 74 (1995) 4384; F. Sartogo, Ph. D. thesis, Università di Roma La Sapienza, Roma, 1994, unpublished. 